\title{
DIE FÖRDERUNG LITERARISCHER KOMPETENZEN IM DAF-UNTERRICHT: HERAUSFORDERUNGEN IN DER PRAXIS
}

\author{
Mojca Leskovec" \\ Filozofska fakulteta, Univerza v Ljubljani
}

\begin{abstract}
Sowohl traditionell als auch mit der Einführung des Gemeinsamen Europäischen Referenzrahmens für Sprachen (GER) spielt(e) Literatur im Deutsch-als-Fremdsprache-Unterricht eine geringe Rolle. In der Fachliteratur werden als Gründe dafür die Tatsachen angeführt, dass Literatur selbst eine Fremdsprache, zugleich aber auch ein Freiraum ist und ihr Rezipieren schwer messbar ist. Eine Befragung unter gymnasialen DaF-Lehrkräften in Slowenien hat gezeigt, dass die Mehrheit der Teilnehmenden literarische Texte in ihrem DaF-Unterricht nur manchmal behandelt, $u$. $z w$. vor allem auf höheren Niveaustufen. Als Gründe dafür führten sie in erster Linie Zeitmangel und mangelhafte Sprachkenntnisse der SchülerInnen an.
\end{abstract}

Schlüsselwörter: Deutsch als Fremdsprache, interkulturelle Kompetenzen, literarische Texte, Literaturdidaktik.

\section{EINFÜHRUNG}

Im Vergleich zum Deutsch-als-Erstsprache-Unterricht spielt(e) Literatur im Deutsch-als-Fremdsprache-Unterricht sowohl traditionell als auch mit der Einführung des Gemeinsamen Europäischen Referenzrahmens für Sprachen (GER) eine geringere Rolle. In der Fachliteratur werden als Gründe dafür die Tatsachen angeführt, dass Literatur selbst eine Fremdsprache, zugleich aber auch ein Freiraum ist und ihr Rezipieren schwer messbar ist (vgl. Euba und Warner, 2017; Šlibar, 2011). Darüber hinaus sei die Auseinandersetzung mit literarischen Texten nicht ganz im Sinne der Handlungsorientierung, auf die der moderne Fremdsprachenunterricht ausgerichtet ist (vgl. Dobstadt und Riedner, 2017). Doch der Fremdsprachenunterricht der Postmethodenära beruht auf einer Reihe von didaktisch-methodischen Prinzipien, und auch wenn Literatur einerseits nicht ganz im Sinne des didaktisch-methodischen Prinzips der Effizienzorientierung ${ }^{1}$ sein sollte, so dürfte andererseits ihr

\footnotetext{
mojca.leskovec@ff.uni-lj.si

1 Die Effizienzorientierung steht einerseits mit dem Prinzip der (Zeit)Ökonomie und andererseits mit der Erfolgsbestätigung und -sicherung zusammen. S. Anm. 2.
} 
Beitrag zur interkulturellen Orientierung als einem weiteren Prinzip für den fremdsprachlichen Unterricht nicht unerwähnt bleiben. ${ }^{2}$

DeCillia und Klippel(2016:630) stellen drei Phasen im Einsatz literarischer Texte im Fremdsprachenunterricht fest: seit 1945 bis in die 1970er Jahre als Periode der intensiven Lektüre literarischer Texte im fortgeschrittenen Unterricht, worauf eine Periode der Bevorzugung von Sachtexten und journalistischer Prosa folgte. Der danach, in den 80er Jahren, aufkommenden rezeptionsästhetischen und interkulturellen Literaturdidaktik hat man laut Dobstadt und Riedner (2017) zu verdanken, dass Literatur im Fremdsprachenunterricht dennoch verankert blieb - allerdings als Gesprächsanlass und Träger von landeskundlichen Informationen, während ihr Potenzial für sprachliches und kulturbezogenes Lernen unausgeschöpft blieb. Euba und Warner (2017: 7) kann man unbestreitbar zustimmen, dass „seit dem Erscheinen des Europäischen Referenzrahmens für Sprachen (2001) /.../ literarische Texte in den Lehrwerken für Deutsch als Fremdsprache und Zweitsprache eine immer geringere Rolle /spielten/“. Der GER weiß nämlich, wie Dobstadt und Riedner (2017) konstatieren, „mit der ästhetischen Sprachverwendung kaum etwas anzufangen". Es stellt sich folglich die Frage, inwieweit literarische Texte in den DaF-Unterricht integriert sind.

Dieser Beitrag setzt sich zweierlei zur Aufgabe: erstens, den Stellenwert von Literatur im Fremdsprachenunterricht der Postmethodenära kurz zu skizzieren, und zweitens, am Beispiel der DaF-Lehrkräfte in Slowenien mithilfe einer schriftlichen Befragung $\mathrm{zu}$ erfahren, wie oft literarische Texte im gymnasialen DaF-Unterricht behandelt werden, was dabei die Orientierung darstellt, welche Aktivitäten zur Förderung der literarischen Kompetenzen eingesetzt werden und mit welchen Schwierigkeiten sich die Lehrenden konfrontiert sehen. Es werden zunächst wichtige Aspekte aus den Fachdiskussionen zum Thema geschildert. Des Weiteren wird die Rolle der Literatur im aktuellen slowenischen Nationalkurrikulum für Deutsch als

\footnotetext{
${ }^{2}$ Es bestehen unterschiedliche Auflistungen von didaktisch-methodischen Prinzipien bzw. Unterrichtsprinzipien. Als die Unterrichtsprinzipien des heutigen (Fremd)Sprachenunterrichts stellt Klippel (2016: 317-319) die Kompetenz- und Handlungsorientierung, Selbsttätigkeit und Authentizität heraus. Grotjahn und Kleppin (2013: 26) führen neben der Handlungs- und Kompetenzorientierung sowie der Lernerorientierung und -aktivierung die Interaktionsorientierung, Förderung von autonomem Lernen, interkulturelle Orientierung, Mehrsprachigkeits- und Aufgabenorientierung an. Von der Fort- und Weiterbildungsreihe Deutsch lehren lernen des Goethe-Instituts (2013) werden für die Deutschvermittlung insgesamt zehn didaktisch-methodische Prinzipien empfohlen: Kompetenzorientierung, Lernerorientierung, Handlungsorientierung, Lerneraktivierung, Interaktionsorientierung, Förderung von Lernerautonomie, interkulturelle Orientierung, Aufgabenorientierung, Mehrsprachigkeitsorientierung sowie Integration digitaler Medien. Kosevski Puljić (2015: 15-17) unterscheidet zwischen didaktischen und methodischen Prinzipien und führt in Bezug auf die ersten folgende an: Aktivierung, Differenzierung/Individualisierung, Erfolgsbestätigung und -sicherung, Elementarisierung, Ganzheit, Handlungsorientierung, Lebensnähe, Motivierung, Ökonomie, Realitätsbezogenheit, Sachgemäßheit, Schülerorientierung, Strukturierung, Veranschaulichung, Zielgemäßheit sowie Interdisziplinarität/fächerübergreifender Fachunterricht.
} 
Fremdspracheam Gymnasium sowieim Abiturkatalog dargestellt.Schließlich folgt der empirische Teil mit Darstellung der Forschungsergebnisse.

\section{STELLENWERT VON LITERATUR IM FREMDSPRACHENUNTERRICHT}

Euba und Warner (2017: 7) sehen den Grund für den höheren Stellenwert von Literatur im Deutsch-als-Erstsprache-Unterricht als im DaF-Unterricht im ihr zugeschriebenen allgemeinen Bildungswert, für den man im effizienzorientierten Fremdsprachenunterricht keinen Platz sah. Diesen hohen Bildungswert erkennt im Kapitel über ästhetische Sprachverwendung allerdings auch der GER (2001). Lovrović und Kolega (2018: 269), die es gerade wegen ihrer humanistischen und erzieherischen Rolle für essenziell halten, dass Lernende bereits in den frühen Phasen des Fremdsprachenunterrichtes mit dem Lesen literarischer Texte beginnen, erwähnen dennoch eine weitere mögliche Ursache dafür, dass in der Geschichte der fremdsprachlichen Didaktik die Einbeziehung der Literatur unterschätzt wurde, und zwar, dass sich die Lehrenden dafür oft inkompetent fühl(t)en.

Dobstadt und Riedner (2017) erkennen in der heutigen Situation widersprüchliche Tendenzen: einerseits kommt Literatur in den aktuellen DaF-Lehrwerken nur noch als Gesprächsanlass vor, während die aufgrund möglicher Stereotypisierungen problematische interkulturelle Perspektive ${ }^{3}$ verschwunden ist, andererseits formiert sich eine Gegenbewegung, die der Literatur im Fremdsprachenunterricht einen höheren Stellenwert zuerkennt, „und zwar nicht trotz, sondern wegen ihrer ästhetischen Dimension“, wobei für die Entwicklung der Kramschen symbolischen Kompetenz ${ }^{4}$ plädiert wird (ebd.). Laut Schweiger (2015: 23) ist für den Einsatz von Literatur im Fremdsprachenunterricht entscheidend, „ihr spezifisches Potenzial zu nutzen und ihre Form mitzureflektieren, um damit die Sprache selbst und ihre unterschiedlichen Aussagemodi und Ausdrucksmöglichkeiten zum Thema und zum Gegenstand des Unterrichts zu machen". So können literarische Texte für kulturreflexives Lernen genutzt werden, denn Kulturreflexion ist auch Sprachreflexion und umgekehrt (Dobstadt und Riedner, 2017).

Lütge (2016: 456) betont die Bedeutung der literarischen Texte für den Erwerb interkultureller Kompetenzen, „weil sie die kulturelle Komplexität mit ihrer Vielzahl von Diskursen und Charakteren sowie möglichen Konflikten darstellen und zwischen dem spannungsreichen Verhältnis von individuellen und kollektiven Identitäten zu vermitteln vermögen", während Hallet (2016: 41) darauf hinweist, dass sie „schon allein deshalb

${ }^{3}$ Laut Dobstadt und Riedner (2017) barg der Kulturvergleich die Gefahr, »Stereotypisierungen zu verfestigen anstatt sie aufzubrechen «.

${ }^{4}$ Laut Kramsch (2011a, in Kramsch und Whiteside, 2015) ist die symbolische Kompetenz ,the ability to manipulate the three dimensions of language as a symbolic system: symbolic representation, symbolic action, symbolic power". 
ihren Platz im Fremdsprachenunterricht/haben/, weil sie selbst authentische kulturelle Äußerungen darstellen“. Anstatt Stereotype und Vorurteile zu produzieren oder zu vertiefen, können literarische Texte dazu anregen, sich mit ihnen auseinanderzusetzen, schreibt Schweiger (2015: 22). Eine wichtige Rolle dabei spielen die Lehrenden.

\subsection{Herausforderungen des Literaturunterrichts}

Tatsächlich ist und bleibt die Sprache der Literatur, wie es Euba und Warner (2017: 6) formulieren, „auch für muttersprachliche Leser/innen immer auch ein Stück Fremdsprache". Doch andererseits haben gerade literarische Texte eine affektive und emotionale Wirkung "und bilden auf diese Weise eine Brücke zwischen der fremdsprachigen fiktionalen Welt und der Erfahrungswelt der Lesenden", stellt Hallet (2016: 42) fest. Somit ist Literatur als fremde Sprache auf einem bestimmten Niveau mit der Muttersprache verwandt (Kosevski Puljić, 2015: 159).

Seit den 80er Jahren des 20. Jahrhunderts ist der Umgang mit literarischen Texten im Deutschunterricht „,stark von der Rezeptionsästhetik geprägt, die die Deutungsoffenheit der Literatur betont und die Lesenden - die hier zugleich auch Lernende sind - den Freiraum gibt, sich kreativ und (je nach literaturdidaktischem Ansatz auch) produktiv mit literarischen Texten auseinanderzusetzen" (Euba und Warner, 2017: 8). Dass Literatur ein Freiraum ist, ist auch nach Šlibar (2011: 54) vermutlich einer der Gründe, „warum man/frau sich bisher nicht so genau und systematisch damit beschäftigt hat, was sich denn da in der Klasse tut, wenn ein literarischer Text ,behandelt' wird“. Wie sie allerdings mehrmals hervorhebt, kann und soll das Erleben und Erfahren von Literatur nicht gemessen werden. Es kann zwar gefördert, erweitert und vertieft werden, doch das Einzige, was bewertet und gemessen werden kann, sind „Fähigkeiten, sich über literarische Texte (Kunst) zu äußern“ (ebd.: 55-56). Dabei soll man im Auge behalten, dass „die Wirkungen von Literatur langfristig, oft nicht konkret und direkt erfassbar sind" (ebd.).

Ein weiterer seitens der Lehrenden nicht selten vorkommender Vorwurf gegen Literatur im Fremdsprachenunterricht sind die sprachlichen Defizite der Lernenden. Auch dieser wäre laut Bonnet und Decke-Cornill (2016: 160) als unberechtigt zurückzuweisen, denn

/d/ie Auffassung, inhaltlich komplexe Aushandlungen seien sprachlich von Lernenden in den ersten Jahren ihres Fremdsprachenerwerbs nicht zu leisten, ist zum einen nicht gesichert und müsste im Einzelfall nachgewiesen werden; zum zweiten wäre der Umgang mit ihren Sprachdefiziten eine Gelegenheit für die Schülerinnen und Schüler, sich in Kompensationsstrategien einzuüben; und drittens wäre eine auf Deutsch /in der Erstsprache/ geführte Diskussion eine weitere Option. 
Lovrović und Kolega (2018: 271) weisen darauf hin, dass nur literarische Texte, die sprachlich und inhaltlich dem Alter und Vorwissen der Lernenden gerecht sind, auch motivierend sein können. Šlibar (2011:94) weist in diesem Zusammenhang allerdings darauf hin, dass sprachlich zugängliche Texte nicht unbedingt auch literarisch einfach sind: „Ein leichter Zugang zum Text kann häufig eben irreführend sein; manchmal haben die Lernenden weniger Zugangsprobleme als die Lehrenden, weil sie weniger vorbelastet sind." Und letztendlich ist auch die Tatsache, dass literarische Texte für die Lernenden schwer zu bearbeiten sein könnten, nach Lovrović und Kolega (2018: 274) kein Argument dafür, sie im Fremdsprachenunterricht nicht zu behandeln. Šlibar (2011: 82) empfiehlt allerdings die Verwendung möglichst vieler innovativer Methoden, ",die vor allem die Selbständigkeit der Lernenden fördern und ihren Dialog untereinander".

\section{LITERATUR IM SLOWENISCHEN NATIONALKURRIKULUM UND ABITURKATALOG ${ }^{5}$}

Nach dem aktuellen slowenischen Nationalkurrikulum für Deutsch als Fremdsprache am Gymnasium sind literarische Texte Teil des Deutschunterrichts und der Ausgangspunkt für die Entwicklung der sprachlichen, literarischen und interkulturellen Kompetenz (Holc et al., 2008: 17). ${ }^{6}$ Das Lesen literarischer Texte wird dabei als Lesen authentischer Texte und damit als das (notwendige) Gegengewicht zu den (oft) nicht authentischen Lehrwerkstexten verstanden (ebd.). Durch literarische Texte entwickeln die SchülerInnen nach Holc et al. (ebd.: 18) folgende Fähigkeiten: Unterscheiden zwischen Realität und Fiktion, Interpretieren, Lesegenuss und -freude, Verstehen der übertragenen Bedeutung und des symbolischen Sprachgebrauches, Zulassung und Verständnis der Fremdheit und Andersartigkeit eines literarischen Textes, Empathie, Annehmen verschiedener Auffassungen und Interpretationen, eigene Produktion sowie Bewerten literarischer Texte.

Nach dem Abiturkatalog wird bei der Behandlung von Literatur im DaF-Unterricht vom Lesen des vollständigen Textes ausgegangen (Gliha Olenik et al., 2017: 33). Vorgesehen ist das Verstehen des Textes in all seinen Dimensionen; ein derartiges Verstehen verlangt einerseits die Einordnung in die deutschsprachige literarische Tradition, andererseits die Berücksichtigung

\footnotetext{
${ }^{5}$ Der jeweilige Abiturkatalog für Deutsch beruht auf dem aktuellen Nationalkurrikulum für DaF am Gymnasium und enthält Informationen über Prüfungsziele, Aufbau und Bewertung der Prüfung, Prüfungsinhalte sowie Beispiele der Prüfungsaufgaben.

${ }^{6}$ Šlibar (2011: 57) spricht „aus praktischen, anschaulichen und analytischen Gründen“ über literarische Kompetenzen. Sie unterscheidet zwischen folgenden: systemische Kompetenzen, funktionale Kompetenzen, strukturelle Kompetenzen, Rezeptions- u. Verarbeitungskompetenzen, diskursive - metaliterarische - reflexive Kompetenzen, inter/kulturelle Kompetenzen, produktive Kompetenz (ebd.: 60-72).
} 
der persönlichen Erfahrung des Lernenden, die auf sein interkulturelles Lesen Einfluss nimmt (ebd.). Auf dem höheren Niveau der Abiturprüfung (B2) soll der Kandidat/die Kandidatin zusammenhängende Texte schreiben können, in denen er/sie das Kennen des vollständigen literarischen Textes zeigt, auf Grundlage dessen er/sie bestimmte Standpunkte beschreibt, verteidigt bzw. zurückweist sowie die eigene Meinung vorstellt; ebenso soll der Kandidat/die Kandidatin die vorgeschriebenen literarischen Texte mündlich beschreiben und interpretieren können (Gliha Olenik et al., 2017: 6).

\section{EMPIRISCHER TEIL}

\subsection{Forschungsproblem}

Nach dem aktuellen slowenischen Nationalkurrikulum für Deutsch als Fremdsprache ${ }^{8}$ am Gymnasium sind literarische Texte Teil des Deutschunterrichts und der Ausgangspunkt für die Entwicklung der sprachlichen, literarischen und interkulturellen Kompetenz(en). Weitere Richtlinien gibt der jeweilige Abiturkatalog vor, der literarische Texte in Anlehnung an den GER allerdings erst auf dem höheren Niveau der Abiturprüfung (B2) vorschreibt. Es stellt sich folglich die Frage, inwieweit literarische Texte in den DaF-Unterricht an slowenischen Gymnasien, der mehrheitlich nicht weit über das Niveau B1 hinausreicht, integriert sind.

\subsection{Methode und Instrument}

Wir bedienten uns der quantitativen Forschung und haben eine schriftliche Befragung der Lehrkräfte vorgesehen, für die ein Fragebogen mit drei geschlossenen und drei offenen Fragen sowie drei zusätzlichen Fragen zu demografischen Daten (Geschlecht, Dauer und Art der Berufserfahrung) entwickelt wurde. Die vorgesehene Zeit zum Ausfüllen des Fragebogens war fünf Minuten.

\subsection{Datenerhebung und Datenaufbereitung}

Der Fragebogen wurde an gymnasiale DaF-Lehrkräfte in Slowenien am 21. August 2018 an ihrem jährlichen Studientreffen verteilt. Die Daten aus den Fragebogen wurden in Excel übertragen und mithilfe der deskriptivstatistischen Verfahren analysiert, wobei die Mittelwerte (MT), Standardabweichungen (STD) und Prozentzahlen (f \%) berechnet wurden.

\footnotetext{
${ }^{7}$ Die Forschungsergebnisse wurden bereits im Rahmen der 12. internationalen SOEGV-Tagung am 6. November 2019 in Maribor vorgestellt.

${ }^{8}$ Für Deutsch als erste Fremdsprache für Fortgeschrittene, als zweite Fremsprache für Fortgeschrittene sowie Anfänger und für Deutsch als dritte Fremdsprache.
} 


\subsection{Teilnehmende}

An der Befragung nahmen 37 Deutschlehrerinnen aus Slowenien teil. Dabei unterrichteten alle außer einer, die als Schulart Mittelschule angegeben hat, an Gymnasien. Die Stichprobe $(\mathrm{N}=37)$ ist im Allgemeinen klein, so dass die Ergebnisse nicht auf die Gesamtpopulation der Deutschlehrenden in Slowenien verallgemeinerbar sind, doch wenn man bedenkt, dass es in Slowenien insgesamt lediglich etwa 60 Gymnasien gibt, ermöglichen sie doch einen Einblick in die vorherrschende Praxis und erscheinen übertragbar auf reale Lehr-Lern-Kontexte zu sein.

Tabelle 1 zeigt, dass die teilnehmenden Lehrerinnen durchschnittlich über 20,8 Jahre Berufserfahrung verfügen. Eine Teilnehmende hatte $\mathrm{zu}$ dem Zeitpunkt erst seit zwei Jahren unterrichtet, eine weitere bereits seit 35 Jahren.

Tabelle 1: Berufserfahrung der Teilnehmenden

\begin{tabular}{|c|c|c|c|c|}
\hline \multicolumn{5}{|c|}{ Wie lange unterrichten Sie schon DaF? } \\
\hline N & MT & STD & MIN & MAX \\
\hline 37 & 20,8 & 8,3 & 2 & 35 \\
\hline
\end{tabular}

\subsection{Forschungsergebnisse und Diskussion}

Mit der ersten geschlossenen Frage wollten wir herausfinden, wie oft Lehrende im DaF-Unterricht literarische Texte behandeln. Tabelle 2 kann entnommen werden, dass nur etwa elf Prozent von ihnen regelmäßig literarische Texte in ihrem Unterricht behandeln. Darunter waren lediglich Lehrende mit mehr als 25 Jahren Berufserfahrung. Nur eine Teilnehmende hat angegeben, dass sie literarische Texte in ihrem Unterricht oft behandelt, eine weitere hat die Antwort nie gewählt. Die Mehrheit hat sich mit fast $84 \%$ allerdings für die Antwort manchmal entschieden.

Tabelle 2: Häufigkeit der Behandlung von Literatur im DaF-Unterricht

\begin{tabular}{|l|l|c|c|}
\hline Fr. $\mathbf{1}$ & Wie oft behandeln Sie im DaF-Unterricht literarische Texte? & F & f \% \\
\hline c) & manchmal & 31 & 83,8 \\
\hline a) & regelmäßig & 4 & 10,8 \\
\hline b) & oft & 1 & 2,7 \\
\hline d) & nie & 1 & 2,7 \\
\hline Gesamt & 37 & 100,0 \\
\hline
\end{tabular}

Mit der zweiten geschlossenen Frage wollten wir feststellen, in welchen Klassen die Teilnehmenden literarische Texte behandeln. Tabelle 3 zeigt, dass die Antworten ziemlich gleichmäßig verteilt sind: 13 Teilnehmende behandeln literarische Texte zwar nur bei der Abiturvorbereitung, doch jeweils 12 in allen Klassen bzw. nur bei Fortgeschrittenen. Für die Antwort nirgendwo hat sich hier keine entschieden. 
Tabelle 3: Behandlung der Literatur nach Unterrichtsstufe

\begin{tabular}{|l|l|c|c|}
\hline Fr. 2 & In welchen Klassen behandeln Sie literarische Texte? & F & f \% \\
\hline c) & nur bei der Abiturvorbereitung & 13 & 35,2 \\
\hline a) & in allen Klassen & 12 & 32,4 \\
\hline b) & nur bei Fortgeschrittenen & 12 & 32,4 \\
\hline d) & in keiner Klasse & 0 & 0,0 \\
\hline Gesamt & 37 & 100,0 \\
\hline
\end{tabular}

Aus der Tatsache, dass sich die Gruppen Abiturvorbereitung und Fortgeschrittene nahe sind und sich decken können, kann man schließen, dass etwa zwei Drittel der Teilnehmenden literarische Texte nur auf höheren Niveaustufen einführen, während ein Drittel der Teilnehmenden literarische Texte in allen Klassen behandelt.

Mit der dritten geschlossenen Frage wollten wir erfahren, an welchem Dokument sich die Lehrenden bei der Einbeziehung der Literatur in ihren Unterricht hauptsächlich orientieren. Aus Tabelle 4 ist ersichtlich, dass sich fast eine Hälfte der Teilnehmenden an dem jeweils gültigen Abiturkatalog orientiert und fast ein Viertel an dem benutzten Lehrwerk. Für die Antwort das Nationalkurrikulum hat sich 18,9\% der Teilnehmenden entschieden und für die Antwort der Gemeinsame Europäische Referenzrahmen 5,4 \%.

Tabelle 4: Einbeziehung der Literatur und Orientierung an Dokumenten

\begin{tabular}{|l|l|c|c|}
\hline Fr. $\mathbf{3}$ & $\begin{array}{l}\text { Was bestimmt in erster Linie die Einbeziehung von Literatur in } \\
\text { Ihren DaF-Unterricht? }\end{array}$ & F & $\mathbf{f}$ \% \\
\hline c) & der Maturakatalog & 18 & 48,7 \\
\hline d) & das benutzte Lehrwerk & 9 & 24,3 \\
\hline b) & das Nationalkurrikulum & 7 & 18,9 \\
\hline a) & der Gemeinsame Europäische Referenzrahmen & 2 & 5,4 \\
\hline Gesamt & 36 & 97,3 \\
\hline
\end{tabular}

Der hohe Anteil der Antworten der Maturakatalog ${ }^{9}$ hängt vermutlich mit der Tatsache zusammen, dass dieser genauere Richtlinien als das Nationalkurrikulum vorgibt. Auch die Häufigkeit der Antwort das benutzte Lehrwerk überrascht einen nicht, wenn man bedenkt, dass an öffentlichen Schulen lediglich vom Schulamt zugelassene Lehrwerke gebraucht werden dürfen, die mit dem Nationalkurrikulum übereinstimmen. Dass sich für die Antwort der Gemeinsame Europäische Referenzrahmen lediglich zwei Teilnehmende entschieden haben, ist wahrscheinlich die Folge davon, dass im GER nur wenige Richtlinien bezüglich der Einbeziehung von Literatur in den Unterricht zu finden sind.

\footnotetext{
${ }^{9}$ Im Slowenischen heißt das Abitur matura und weil dieser Ausdruck auch in einem Teil des deutschsprachigen Raumes existiert, wurde in der Umfrage die unter slowenischen Deutschlehrkräften verbreitete Benennung Maturakatalog benutzt.
} 
Was jedoch auffällt, ist die Tatsache, dass alle, die sich bei der dritten Frage für die Antwort das Nationalkurrikulum entschieden haben, bei der ersten Frage angegeben haben, dass sie literarische Texte in ihrem DaFUnterricht (nur) manchmal behandeln. Das Nationalkurrikulum geht nämlich davon aus, dass literarische Texte Teil des Deutschunterrichts und der Ausgangspunkt für die Entwicklung der sprachlichen, literarischen und interkulturellen Kompetenz(en) sind. Der Grund könnte darin liegen, dass es keine genaueren Angaben über den Anteil der Literatur im gymnasialen DaF-Unterricht vorgibt, obwohl es den Ansatz, die Kompetenzen und Ziele beschreibt.

Mit der vierten offenen Frage wollten wir herausfinden, was für literarische Texte die Lehrenden in ihrem DaF-Unterricht behandeln. Die Antworten wurden in fünf Kategorien verteilt. Ihre Häufigkeit ist aus der Tabelle 5 ersichtlich.

Tabelle 5: Anteil einzelner Literaturgattungen im DaF-Unterricht

\begin{tabular}{|l|l|c|c|}
\hline Fr. 4 & Was für literarische Texte behandeln Sie im DaF-Unterricht? & F & f \% \\
\hline 4.1 & Lyrik & 29 & 78,4 \\
\hline 4.2 & Prosa & 20 & 54,1 \\
\hline 4.3 & anderes & 8 & 21,6 \\
\hline 4.4 & Abiturlektüre & 7 & 18,9 \\
\hline 4.5 & Dramatik & 1 & 2,7 \\
\hline
\end{tabular}

Mit 78,4 \% haben die meisten Teilnehmenden Lyrik angegeben, gefolgt von Prosa mit 54,1\%. Acht Teilnehmende haben unterschiedliche andere Antworten angeführt, u. zw. Ausschnitte aus der Jugendliteratur; Auszüge aus literarischen Texten; zweisprachige Texte; Texte, mit deren Thema sich die Schüler auseinandersetzen können; kurze Texte, die zu bestimmten Themen passen. Sieben Teilnehmende haben die Abiturlektüre angegeben und nur eine Dramatik.

Die Frage nach der Literaturgattung wurde absichtlich etwas breiter gestellt, so dass sich die Teilnehmenden beim Antworten nicht eingeschränkt fühlen würden und wir möglichst viele unterschiedliche Antworten, eventuell mit genaueren Beschreibungen, sammeln könnten, die uns bei der weiteren Forschung behilflich wären. Dass den ersten Platz unter den Antworten gerade Lyrik einnimmt, ließe sich im Kontext anderer Antworten aus der Befragung mithilfe der Zeitökonomie interpretieren, denn Lyrik ist häufig wesentlich kürzer als Prosa, die zwar auch vorkommt, doch wie in der Kategorie anders hervorgehoben wird, lieber in Form von kurzen Ausschnitten. Die Abiturlektüre besteht sowohl aus Prosa als auch aus Lyrik und war eine erwartete Antwort - auch in Hinsicht auf die sehr häufige Angabe der Orientierung am Abiturkatalog. Dass die Antwort Dramatik nur einmal vorkommt, hängt vermutlich mit der Abiturlektüre zusammen, die fast ausschließlich aus den beiden anderen Literaturgattungen besteht. 
Mit der fünften offenen Frage wollten wir die Aktivitäten feststellen, die die Lehrenden zur Förderung der literarischen Kompetenz(en) einsetzen. Am häufigsten ist dabei die Antwort (kreatives) Schreiben vorgekommen. Insgesamt ebenso zu 35,1\% sind andere Antworten vorgekommen, u. zw. etwas auswendig Lernen; Assoziationen und Erwartungen (Vorentlastung); Ideen sammeln; mit der Übersetzung vergleichen; Wortschatzarbeit. Auf Platz drei befinden sich mit 29,7 \% die Fertigkeiten Lesen, Hören, Sehen, gefolgt von Gesprächen und Diskussionen sowie Interpretieren mit jeweils 24,3 \%. Sechsmal ist die Antwort Ausschnitte vorführen vorgekommen und zweimal die Antwort Referate, Präsentationen.

Tabelle 6: Verwendete Aktivitäten zur Förderung der literarischen Kompetenz

\begin{tabular}{|l|l|c|c|}
\hline Fr. 5 & $\begin{array}{l}\text { Was für Aktivitäten setzen Sie zur Förderung der literarischen } \\
\text { Kompetenz ein? }\end{array}$ & F & $\mathbf{f} \%$ \\
\hline 5.1 & (kreatives) Schreiben & 13 & 35,1 \\
\hline 5.2 & anderes & 13 & 35,1 \\
\hline 5.3 & Lesen/Hören/Sehen & 11 & 29,7 \\
\hline 5.4 & Gespräche, Diskussionen & 9 & 24,3 \\
\hline 5.5 & Interpretieren & 9 & 24,3 \\
\hline 5.6 & Ausschnitte vorführen & 6 & 16,2 \\
\hline 5.7 & Referate, Präsentationen & 2 & 5,4 \\
\hline
\end{tabular}

Interessanterweise ist am häufigsten die Antwort (kreatives) Schreiben vorgekommen, also fördern die Lehrenden demnach in erster Linie eine produktive Auseinandersetzung mit literarischen Texten. Nur teilweise kann dies auch damit zusammenhängen, dass auf dem höheren Niveau der Abiturprüfung ein literarischer Essay erwartet wird. Ein weiteres überraschendes Ergebnis ist die Platzierung der Antwort Referate, Präsentationen, die nur zweimal vorgekommen ist, obwohl dies ein fester Teil des traditionellen Sprachenunterrichtes auf höheren Niveaustufen war.

Mit der letzten, sechsten offenen Frage wollten wir erfragen, mit welchen Schwierigkeiten sich die Lehrenden bei der Förderung der literarischen Kompetenz(en) in der Schule konfrontiert sehen. Am häufigsten - vierzehnmal - haben die Lehrenden dabei den Zeitmangel angeführt, wobei es die meisten nicht näher erklärt haben - lediglich zwei Teilnehmende haben dies durch den Stundeplan begründet, der ihnen keine Doppelstunden ermöglicht, die man aber hierfür ihrer Meinung nach brauchen würde, denn eine Unterrichtsstunde von 45 Minuten sei zu wenig. Sehr häufig war mit 35,1 \% auch die Antwort mangelhafte Sprachkenntnisse der SchülerInnen. Achtmal wurde als Schwierigkeit die Motivation der SchülerInnen angegeben und sechsmal die eigene Unsicherheit. Einmal wurde geringes Vorwissen der SchülerInnen angeführt. Darüber hinaus sind dreimal unterschiedliche andere Faktoren vorgekommen, $\mathrm{u}$. zw. heterogene 
Lerngruppen; zu umfangreicher Lehrplan; nicht mit dem Lehrplan verbunden, geringe Rolle der Literatur für den DaF-Unterricht.

Tabelle 7: Schwierigkeiten bei der Förderung der literarischen Kompetenz in der Schule

\begin{tabular}{|l|l|c|c|}
\hline Fr. 6 & $\begin{array}{l}\text { Was stellt für Sie Schwierigkeiten bei Förderung der } \\
\text { literarischen Kompetenz in der Schule dar? }\end{array}$ & F & f \% \\
\hline 6.1 & Zeitmangel & 14 & 37,8 \\
\hline 6.2 & mangelhafte Sprachkenntnisse der SchülerInnen & 13 & 35,1 \\
\hline 6.3 & Motivation der SchülerInnen & 8 & 21,6 \\
\hline 6.4 & eigene Unsicherheit & 6 & 16,2 \\
\hline 6.5 & anderes & 3 & 8,1 \\
\hline 6.6 & geringes Vorwissen der SchülerInnen & 1 & 2,7 \\
\hline
\end{tabular}

Die am häufigsten angeführte Schwierigkeit, Zeitmangel, ist nicht überraschend, denn Lehrende beschweren sich oft über die große Menge des zu bewältigenden Lernstoffes. Vermutlich hängt sie auch mit der Effizienzorientierung des fremdsprachlichen Unterrichts zusammen. Auch die der Häufigkeit nach folgende Antwort mangelhafte Sprachkenntnisse der SchülerInnen erscheint nicht unerwartet. Die Antworten Motivation der SchülerInnen und insbesondere eigene Unsicherheit mit 21,6 bzw. 16,2 \% hingegen tauchen weniger erwartet auf. Im Rahmen ihres Studiums setzen sich die angehenden DaF-Lehrkräfte sowohl an der Universität Ljubljana als auch an der Universität Maribor nämlich in mehreren Lehrveranstaltungen eingehend mit Literatur sowie Literaturdidaktik auseinander. Es lässt sich dabei auch kein Zusammenhang dieser Antworten mit beispielsweise der Berufserfahrung feststellen. Einer der möglichen Gründe dafür wäre eventuell die von der Rezeptionsästhetik stammende Deutungsoffenheit der Literatur. Um festzustellen, inwieweit dies mit der eigenen Vorliebe der Lehrenden für Literatur zusammenhängt, wäre allerdings eine weitere (Um) Frage erforderlich. Unverständlich ist jedoch die Angabe, dass Literatur nicht mit dem Lehrplan verbunden ist; wie oben angeführt versteht nämlich der Lehrplan Literatur stets als Teil des gymnasialen DaF-Unterrichts.

\section{SCHLUSSBEMERKUNGEN}

In der vorliegenden Forschung sind wir auf die Frage eingegangen, inwieweit literarische Texte in den DaF-Unterricht an slowenischen Gymnasien integriert sind. Die Forschungsergebnisse stellen einige interessante, teilweise jedoch auch überraschende Aspekte dar. Abschließend kann man zusammenfassen, dass die Mehrheit der teilnehmenden Lehrenden literarische Texte in ihrem DaF-Unterricht manchmal behandelt, u. zw. vor allem auf höheren Niveaustufen. Dabei orientieren sich die Lehrenden an dem Abiturkatalog, der literarische Texte in Anlehnung an den GER erst auf dem höheren Niveau der Abiturprüfung (B2) vorschreibt. Als Schwierigkeiten bei 
Förderung der literarischen Kompetenzen im schulischen DaF-Unterricht führten die Teilnehmenden in erster Linie Zeitmangel und mangelhafte Sprachkenntnisse der SchülerInnen an, gefolgt von (fehlender) Motivation der SchülerInnen und, etwas überraschend, eigener Unsicherheit. Ein weiterer interessanter Aspekt ist die vorherrschende Aktivität, die die Teilnehmenden zur Förderung der literarischen Kompetenzen in ihrem Unterricht einsetzen, nämlich (kreatives) Schreiben $d$. h. eine produktive Auseinandersetzung mit literarischen Texten.

Literatur als - sowohl im Hinblick auf die Entwicklung literarischer und interkultureller Kompetenzen als auch auf den allgemeinen Bildungswert der angehenden AkademikerInnen - äußerst wichtiger Bereich spielt in den aktuellen Lehrwerken für $\mathrm{DaF}$, die sich konsequent am GER orientieren, eine geringe Rolle und wird somit im Allgemeinen tatsächlich zum größten Teil den Lehrenden überlassen, die nun dafür auch selber im Rahmen von Seminaren und Fortbildungen entsprechend sensibilisiert werden sollten. In erster Linie sollten sie dabei das Potenzial der Literatur für den fremdsprachlichen Unterricht (wieder) erkennen, des Weiteren ihre eigene Unsicherheit im Umgang mit Literatur abbauen und selbst zur Auseinandersetzung mit literarischen Texten motiviert werden. Im Anschluss daran sollten sie Methoden kennenlernen oder aber auffrischen, die dabei behilflich sein könnten, ihre SchülerInnen zur Auseinandersetzung mit Literatur zu motivieren.

Das oberste Ziel des Fremdsprachenunterrichts ist unbestreitbar die kommunikative Kompetenz. Zu ihr gehören, wie Bonnet und Decke-Cornill (2016: 157) mehrfach hervorheben, allerdings auch Bildungsziele:

Bezogen auf Sprache sollen die Lernenden deren Funktion für die Konstruktion von Identität und deren Wirkungsweise in unterschiedlichen Diskursen verstehen und bewerten sowie die horizonterweiternden und -überschreitenden Möglichkeiten literarisch-ästhetischer Kommunikation kennenlernen.

Šlibar (2011: 42) definiert das generelle Ziel des Literaturunterrichts folgendermaßen: „,/.../ das bei hochwertiger Literatur auftretende Unbehagen durch genaues Verstehen und produktives Handeln in Vergnügen umzuwandeln". Befolgt man dieses Ziel, so steigt vielleicht auch durch die internationale Grundschul-Lese-Untersuchung PIRLS 2016 festgestellte Zahl der (slowenischen) SchülerInnen, die gerne lesen. Inwieweit literarische Texte in den DaF-Unterricht an slowenischen Grundschulen integriert sind, bedarf allerdings weiterer Forschung. 


\section{LITERATUR}

Bonnet, A. und Decke-Cornill, H. (2016) Inhalte zur Entwicklung sprachlicher und literarischer Kompetenzen. In Burwitz-Melzer, E., Mehlhorn, G., Riemer, C., Bausch, K. und Krumm, H. (Hrsg.) Handbuch Fremdsprachenunterricht (6., völlig überarbeitete und erweiterte Auflage). Tübingen: A. Francke Verlag, 157-162.

De Cillia, R. und Klippel, F. (2016) Geschichte des Fremdsprachenunterrichts in deutschsprachigen Ländern seit 1945. In Burwitz-Melzer, E., Mehlhorn, G., Riemer, C., Bausch, K. und Krumm, H. (Hrsg.) Handbuch Fremdsprachenunterricht (6., völlig überarbeitete und erweiterte Auflage). Tübingen: A. Francke Verlag, 625-631.

Dobstadt, M. und Riedner, R. (2017) Von der Literatur lernen. Magazin Sprache. https://www.goethe.de/de/spr/mag/idt/von.html (28. 1. 2020)

Europarat (2001) Gemeinsamer Europäischer Referenzrahmen für Sprachen: lernen, lehren, beurteilen. https://www.goethe.de/Z/50/commeuro/i0.htm (18. 2. 2018)

Euba, N. und Warner, C. (2017) Literatur Lesen Lernen. In Dobstadt, M. und Riedner, R. (Hrsg.) Literatur Lesen Lernen: Lesewerkstatt Deutsch 2. Stuttgart: Ernst Klett Sprachen.

Gliha Olenik, N. et al. (2017) Nemščina: predmetni izpitni katalog za splošno maturo. Ljubljana: Državni izpitni center. https://www.ric.si/mma/M-NEM-2019/2017083009155511/ (13. 8. 2018)

Goethe-Institut (2013) Didaktisch-methodische Prinzipien. <https://www.goethe.de/resources/files/ pdf157/unterrichtsprinzipien12.pdf> (23. 2. 2018)

Holc, N. et al. (2008) Učni načrt. Nemščina: gimnazija: splošna, klasična, strokovna gimnazija. Ljubljana: Ministrstvo za šolstvo in šport, Zavod RS za šolstvo. http://eportal.mss.edus.si/msswww/programi2010/programi/media/pdf/un_gimnazija/un_nemscina_gimn.pdf (13. 8. 2018)

Grotjahn, R. und Kleppin, K. (2013) Handlungsorientierung, Kompetenzorientierung und weitere Prinzipien. In Ende, K., Grotjahn, R., Kleppin, K. und Mohr, I. (2013) Deutsch lehren lernen 6: Curriculare Vorgaben und Unterrichtsplanung. München: Goethe-Institut, Langenscheidt, 26-32.

Hallet, W. (2016) Fokus: Texte - Medien - Literatur - Kultur. In Burwitz-Melzer, E., Mehlhorn, G., Riemer, C., Bausch, K., Krumm, H. (Hrsg.) Handbuch Fremdsprachenunterricht. (6., völlig überarbeitete und erweiterte Auflage). Tübingen: A. Francke Verlag, 39-43.

Klippel, F. (2016) Didaktische und methodische Prinzipien der Vermittlung. In Burwitz-Melzer, E., Mehlhorn, G., Riemer, C., Bausch, K. und Krumm, H. (Hrsg.) Handbuch Fremdsprachenunterricht (6., völlig überarbeitete und erweiterte Auflage). Tübingen: A. Francke Verlag, 315-320.

Kosevski Puljić, B. (2015) Einführung in Theorie und Praxis der Didaktik Deutsch als Fremdsprache. Ljubljana: Znanstvena založba Filozofske fakultete.

Kramsch, C. und Whiteside, A. (2015) What is symbolic competence and what can we do with it? http:// blc.berkeley.edu/wp-content/uploads/2015/08/kramschSC.pdf (13. 7. 2020)

Lovrović, L. und Kolega, C. (2018) Using Literary Texts for the Development of Foreign Language Learners' Intercultural Competence. Strani jezici 47 (4), 265-277. https://stranijezici.ffzg.unizg. hr/wp-content/uploads/2019/09/strani-jezici-47-4-4.pdf (20.12. 2019)

Lütge, C. (2016) Lehr-/Lernmaterialien und Medien zum Aufbau interkultureller Kompetenzen. In Burwitz-Melzer, E., Mehlhorn, G., Riemer, C., Bausch, K. und Krumm, H. (Hrsg.) Handbuch Fremdsprachenunterricht. (6., völlig überarbeitete und erweiterte Auflage). Tübingen: A. Frankke Verlag, 456-459.

Schweiger, H. (2015) Kulturelles Lernen mit Literatur - von Anfang an. Fremdsprache Deutsch, 52, 22-27. https://www.goethe.de/resources/files/pdf95/auszug_fremdsprachedeutsch_.pdf (28. 1. 2020)

Šlibar, N. (2011) Wie didaktisiere ich literarische Texte? Neue Maturatexte und viele andere im DaF-Unterricht. Ljubljana: Znanstvena založba Filozofske fakultete. 


\section{PROMICANJE KNJIŽEVNIH KOMPETENCIJA U NASTAVI NJEMAČKOGA KAO STRANOG JEZIKA: IZAZOVI U PRAKSI}

U poučavanju je njemačkoga kao stranog jezika književnost, i u tradicionalnom načinu i uvođenjem zajedničkoga europskog referentnog okvira za jezike (ZEROJ), igrala, odnosno trenutačno igra neznatnu ulogu. Stručna literatura kao razlog tome izdvaja činjenicu da je književnost sama po sebi već strani jezik, a istodobno i otvoren prostor te da se njezina recepcija teško može izmjeriti. Istraživanje provedeno među srednjoškolskim nastavnicima njemačkoga kao stranog jezika u Sloveniji pokazuje da se većina sudionika tek ponekad u svojoj nastavi bavi književnim tekstovima, i to prije svega na višim stupnjevima. Kao razloge za to u prvom planu naveli su nedostatak vremena i nedovoljno poznavanje stranoga jezika od strane njihovih učenika.

\section{PROMOTING LITERARY COMPETENCE IN GERMAN AS A FOREIGN LANGUAGE CLASSES: CHALLENGES IN PRACTICE}

Both traditionally and with the introduction of the Common European Framework of Reference for Languages (CEFR), literature has played only a minor role in teaching German as a foreign language. According to the specialist literature, the reason for this lies in the fact that literature is a foreign language in itself; however, at the same time it is also a vast area and its reception is difficult to quantify. A survey conducted among high school teachers of German as a foreign language in Slovenia has shown that the majority of respondents use literary texts in their classes only occasionally and predominantly with higher-level students. The main reasons for this are the lack of time and students' limited language skills.

Keywords: German as a foreign language, intercultural competences, literary texts, didactics of literature.

This work is licensed under a Creative Commons Attribution 4.0 International License.

Ovaj rad dostupan je za upotrebu pod licencom Creative Commons Imenovanje 4.0 međunarodna. 